analecta polit. | Vol. 9 | No. 16 | PP. 37-54 | enero-junio | 2019 | ISSN-e: 2390-0067 (en línea) |

Medellín-Colombia

doi: http://dx.doi.org/10.18566/apolit.v9n16.a03

\title{
Educación, política y universidad
}

\section{Education, politics and university}

\section{Educação, política e universidade}

Cómo citar

este artículo en APA: Díaz, J. A., MuñozBuitrago, D. A. y GarzónPascagaza, E. J (2019). Educación, política y universidad. Analecta Política, 9(16), 37-54.

Fecha de recepción: 24.01.2019

Fecha de aceptación: 14.03.2019

\section{JORGE-AURELIO DÍAZ}

Doctor en Filosofía

Universidad Católica de Colombia

Correo electrónico: jadiaza@ucatolica.edu.co

orcid.org/0000-0001-8576-0815

\section{DARWIN MUÑOZ}

Magíster en Ciencias Políticas

Universidad Santo Tomas

Correo electrónico: darwinmunoz@usantotomas.edu.co orcid.org/0000-0002-3193-6034

\section{EDGAR JAVIER GARZÓN-PASCAGAZA}

Magister en investigación social interdisciplinaria

Universidad Católica de Colombia

Correo electrónico: ejgarzon@ucatolica.edu.co orcid.org/0000-0001-6898-7030 


\section{Resumen}

El texto examina la tarea que debe cumplir la universidad en la formación política de sus estudiantes. Se parte de la función política que ha desempeñado la institución universitaria desde sus mismos orígenes, así como de la importancia de dicha formación para hacer de los futuros profesionales verdaderos ciudadanos de una sociedad democrática. Se señalan los principales propósitos que deberá cumplir dicha formación, así como los escollos que deberán evitarse para que la cátedra docente no termine convirtiéndose en tribuna de proselitismo político.

Palabras clave: educación, política, formación política, ciudadano.

\section{Abstract}

The text examines the task that the university must fulfill in the political education of its students. The analysis starts with the political role that the university institution has played since its very origins, as well as the importance of such training to turn the future professionals into true citizens of a democratic society. The main purposes that this formation will have to fulfill in the future are indicated, as well as the obstacles that should be avoided so that the teaching chair does not end up becoming a tribune of political proselytism.

Keywords: Education, politics, political education, citizen.

\section{Resumo}

O texto examina a tarefa que a universidade deve cumprir na educação política dos seus estudantes. Baseia-se na função política que a instituição universitária tem desempenhado desde suas origens, bem como na importância dessa formação para fazer dos futuros profissionais verdadeiros cidadãos de uma sociedade democrática. Os principais propósitos que esta formação terá que cumprir são indicados, assim como os obstáculos que devem ser evitados para que a aula de ensino não termine se tornando uma tribuna de proselitismo político.

Palavras-chave: Educação, política, formação política, cidadania. 


\section{Introducción}

Inspirados en las reflexiones que hizo el profesor Guillermo Hoyos Vásquez acerca de la labor educativa ${ }^{1}$, nos proponemos retomar una idea que constituyó para él una preocupación constante a lo largo de su labor como docente, pero que no llegó a desarrollar como él hubiera querido hacerlo. En alguna ocasión, en medio de una conversación informal, le escuchamos señalar que le hubiera gustado poder desarrollar un proyecto que le preocupaba, a saber: ¿cómo elaborar, en el seno de la educación superior, una verdadera estrategia para la formación política de los estudiantes que los capacite para el desempeño de sus deberes como ciudadanos, sin que ello signifique convertir la cátedra en una tribuna para el proselitismo político?

No pretendemos, por supuesto, exponer aquí todo un programa que él mismo no tuvo ocasión de desarrollar, a pesar de que, a lo largo de sus diversas intervenciones acerca de la educación, dejó plasmadas abundantes ideas al respecto. Nuestro propósito es mucho más modesto: hemos querido sembrar entre los docentes esa inquietud que tuvo el profesor Hoyos y que, en la coyuntura histórica que nos ha tocado vivir en nuestro país, adquiere una particular significación, porque se trata de una labor que no puede ser realizada por una persona en particular, sino que incumbe a todos los que, de una manera u otra, participamos en las labores académicas en el sistema de educación superior.

Nuestro propósito será, entonces, esbozar algunos conceptos que puedan servir de apoyo para una fructuosa discusión, cuyo objetivo debería ser la elaboración colectiva de ese importante propósito. Debemos tomar conciencia de la importancia del mismo, de los propósitos que deben guiarlo, de los escollos que deberá evitar y de los mejores medios para lograrlo. Se trata de una verdadera tarea colectiva en la que será indispensable contar con el aporte de muy diversas perspectivas, tanto filosóficas como psicológicas, tanto pedagógicas como políticas, y que deberá desarrollarse en el ámbito de la reflexión conceptual, así como también en el de la praxis pedagógica.

Guiados, entonces, por la idea germinal que nos indicó el profesor Hoyos, vamos a tratar de esbozar, primero, algunos conceptos básicos acerca de la impor-

1 Sobre el ideario educativo de Guillermo Hoyos pueden consultarse, entre otros, sus escritos: Filosofía de la educación. Apuntes de su último seminario de doctorado; Ensayos para una teoría discursiva de la educación; Ética comunicativa y educación para la democracia; así como los libros: Guillermo Hoyos: vida y legado y Universidad y sociedad: aproximaciones críticas, tensiones y desafíos, publicados por la Pontificia Universidad Javeriana (ver bibliografía). 
tancia que tiene esta tarea de sembrar en nuestros estudiantes una conciencia clara de sus responsabilidades como ciudadanos de una sociedad democrática; examinaremos luego los propósitos que la misma deberá tener, así como los escollos que deberán evitarse y los medios más idóneos para lograrla. Nuestro propósito, lo repetimos una vez más, no es otro que poner sobre la mesa algunas ideas que podrían ser útiles en el momento de reflexionar sobre el deber que tienen nuestras instituciones académicas de formar no solamente excelentes profesionales en sus diversas áreas del conocimiento, sino verdaderos ciudadanos, conscientes tanto de sus derechos como de sus respectivos deberes.

\section{Importancia de la tarea}

A ningún docente se le puede escapar la importancia que tiene la formación de verdaderos ciudadanos, que sean realmente personas útiles a la sociedad y que estén dispuestos a colaborar, desde sus diversos puestos de trabajo, al bienestar general. Este es, sin lugar a dudas, uno de los principales objetivos de las instituciones de educación superior que nacieron en Europa precisamente cuando la llamada Edad Media había llegado a su madurez, luego de un esfuerzo de varios siglos realizado sobre todo por los monjes para conservar y expandir una cultura que había casi desaparecido. Ya en el siglo IX, cuando Europa comenzaba a reorganizarse, después de la larga y dolorosa desintegración del Imperio romano, el emperador Carlomagno vio la necesidad de establecer escuelas -tanto la Palatina de Aquisgrán, bajo la dirección del famoso Alcuino de York, como las Monacales, en las diversas diócesis y monasterios esparcidos a lo largo del Imperio-. El propósito central de esa tarea era la preparación de los funcionarios que necesitaba el nuevo Estado para su consolidación, así como facilitar la comunicación entre las élites, indispensable para asegurar la legitimidad del poder imperial. Este objetivo se logró, en buena medida, gracias a la unificación lingüística con el llamado "latín medieval", así como con la homogeneización de la escritura mediante la famosa "minúscula carolingia".

Esta vocación política que orientó el llamado "renacimiento carolingio", que tuvo lugar a finales del siglo viII, se hará presente de nuevo cuando, en los siglos XII y XIII, aparezcan las primeras universidades en Europa, comenzando por la más antigua de todas, la de Bolonia, cuyos inicios datan de finales del siglo XI. De manera germinal se organiza, en aquella ciudad del norte de Italia, una primera escuela de derecho; y será precisamente gracias al cultivo del derecho, tanto del 
eclesiástico como del civil, como alcanzará su merecida fama. A su vez, la Iglesia, heredera casi única de la vieja idea de una Europa unificada, vino a ser la patrocinadora de las nuevas universidades, al ver en ellas un elemento de la mayor significación tanto para la organización de la sociedad como para la expansión y consolidación del mensaje religioso.

Ahora bien, si las universidades fueron conscientes, desde sus inicios, de su vocación política, es decir, del papel fundamental que desempeñaban no solamente en la conservación y transmisión de la cultura, sino también en la organización y consolidación de la sociedad, otro tanto cabe decir en sentido inverso, porque todas las sociedades -y muy especialmente las democráticas- necesitan ciudadanos capacitados y conscientes que sepan apreciar los beneficios de una vida en sociedad organizada. Bien puede decirse que las universidades han sido, para la cultura occidental, uno de los baluartes más significativos para su desarrollo y configuración; de modo que entre la sociedad y la universidad se establece lo que bien podemos llamar "círculo virtuoso", en el que cada una contribuye a la consolidación de la otra. Esta labor cobra especial significación como una respuesta al momento actual, en el que, como bien lo ha señalado Martha Nussbaum, "predomina una nueva concepción que gira en torno a la rentabilidad, y que ha dejado de lado la idea del desarrollo personal basado en la imaginación y el pensamiento crítico" (2010, p. 22).

Por lo demás, si pensamos que la política, en el sentido más general de la palabra, se refiere a la forma como en las diversas sociedades se organiza el manejo del poder y de la convivencia, podemos entender muy bien la importancia que tiene el que nuestros estudiantes tomen clara conciencia de lo que significa para todos tanto lo político, en general, como la política, en particular. Imposible pensar que un país pueda llegar a organizarse de manera razonable si sus ciudadanos no disponen de una significativa cultura política; si no cuentan con una comprensión mínima de sus requisitos, exigencias y condiciones de posibilidad, así como de sus limitaciones y debilidades. Y, en el caso de las sociedades democráticas que descansan en lo fundamental sobre la participación activa de los ciudadanos, esa necesidad de que estos últimos dispongan de una suficiente formación política se acrecienta todavía más.

Lo anterior es así porque la política se caracteriza como un saber decisional y el papel que desempeña la educación en el desarrollo de la capacidad decisoria es de suma importancia, en la medida que pone al ser humano en contacto con contenidos cognoscitivos, valorativos y de responsabilidad relacionados con los 


\section{Jorge-Aurelio Díaz, Darwin Muñoz y Edgar Javier Garzón-Pascagaza Educación, política y universidad}

resultados que se puedan alcanzar. Con ello se abre un espacio para que esos resultados, inicialmente entendidos como la simple consecuencia de un conjunto de acciones, se transformen en un verdadero logro, concebido y captado intersubjetivamente como el cumplimiento parcial de un proyecto.

En el proceso de la acción humana, el agente decisor, luego de examinar las posibilidades y de proceder a su análisis crítico, valora, elige y decide aquella línea de conducta de cuyo resultado espera que se obtenga el mayor bien posible. Como bien lo ha señalado Edgar Garzón en referencia al pensamiento de Hannah Arendt, "la acción es reconocida como la categoría de mayor rango, [...] porque es la condición de toda vida política, ya que, en el ejercicio de ella, nos reconocemos como seres humanos [...] y hacemos pleno uso de nuestras competencias" (2017, p. 50). En el desarrollo de dicho proceso, el agente de la decisión asume, o puede asumir al menos, el papel de activador de las potencialidades de convivencia que se hallan presentes en el conjunto de los actores sociales, con lo cual hace posible el avance y la convergencia de todos ellos, para configurar, a través de relaciones de complementación y proyección, un verdadero tejido social.

De modo que, si la universidad nació con una vocación política, y si nuestras sociedades tienen urgente necesidad de que sus ciudadanos dispongan de una sólida cultura política y de una capacidad de decisión conceptualmente elabora$\mathrm{da}$, es preciso que las instituciones universitarias se aperciban de esta importante misión y busquen los medios más adecuados para cumplirla de manera efectiva. Quienes desempeñamos tareas en la academia debemos asumir igualmente nuestra responsabilidad en este sentido y analizar las formas como podemos contribuir de manera eficiente a su cumplimiento, porque, si bien es cierto que la labor que llevan a cabo las universidades en el cumplimiento de sus tareas de docencia, investigación y extensión constituyen un claro servicio a la sociedad, es indispensable, además, que sus egresados adquieran una clara conciencia de sus deberes y de sus derechos como ciudadanos de un Estado de derecho ${ }^{2}$.

2 Los profesores Germán Vargas, Raúl Meléndez y Wilson Herrera han señalado que "una educación filosófica es base de una educación ciudadana, toda vez que pone a los sujetos, a través de su formación, en condiciones de reflexionar sobre sí mismos, sobre su entorno, sobre su relación con los otros" (2017, p. 67). Aunque sus reflexiones se orientan a la enseñanza de la filosofía en el bachillerato, no pocas de ellas pueden aplicarse a la educación política de los estudiantes universitarios. 


\section{Propósitos de la formación política}

Una vez señalada la importancia de la formación política de nuestros estudiantes, veamos algunas reflexiones en torno a los propósitos que debe tener dicha formación.

1. El primero de todos, por supuesto, es que los estudiantes tomen conciencia de la complejidad y riqueza de los fenómenos políticos, y de la responsabilidad que nos incumbe a quienes participamos en la vida pública que nos garantiza un Estado de derecho. Más allá de la participación electoral, todo ciudadano, y en particular aquellos que han alcanzado un nivel superior en la educación, deben disponer de una información básica suficiente que les permita no solamente participar de manera responsable en la elección de los gobernantes, sino también conocer tanto sus derechos como sus deberes, así como la forma adecuada de defender los primeros y cumplir los segundos.

Es que la política es un ámbito de la relación entre personas particularmente intrincado y cambiante, ya que se propone organizar las correlaciones de poder que surgen en el seno de todo grupo humano en el desarrollo de su configuración; correlaciones que se acrecientan y se vuelven cada vez más complejas en la medida que los grupos se hacen más extensos y diversos, y que conducen, por consiguiente, de manera inevitable, al surgimiento de múltiples conflictos. Bien puede decirse que la política es precisamente el arte de manejar los conflictos sociales con exclusión de la violencia.

Lo primero que cabe decir al respecto es que la política, en su sentido más amplio, es decir, lo político, constituye una realidad que no podemos eludir mientras vivamos en sociedad, ya que, como lo ha señalado Hannah Arendt, "[a]l preguntar por el sentido de la política hay una respuesta tan sencilla y tan concluyente en sí misma, que se diría que otras respuestas están totalmente de más. La respuesta es: el sentido de la política es la libertad" (2008, p. 144). Y es a todas luces claro que el ejercicio de la libertad comparte el mismo carácter de la vida, al configurar una fuerza que conecta a todos los miembros de un cuerpo social y determina de manera fundamental su "estado de salud". Pretender escapar a la forma como se organiza y ejerce el poder en el seno de una sociedad y, por consiguiente, a su incidencia en el ejercicio de la libertad, o dejar esa organización y ese ejercicio en manos de unos pocos, no pasa de ser una mera ilusión. Es cierto que las actividades políticas son variadas y exigen una distribución de las tareas y, por consiguiente, una diversidad de compromisos por parte de los ciudadanos, pero, mientras vivamos en sociedad, todos somos, en algún grado, partícipes de esa realidad 


\section{$44 \mid$ Jorge-Aurelio Díaz, Darwin Muñoz y Edgar Javier Garzón-Pascagaza Educación, política y universidad}

insoslayable, tanto de modo activo como pasivo, es decir, tanto cuando actuamos políticamente como cuando nos abstenemos de actuar; de modo que, en vez de pretender escapar a esa realidad, más vale confrontarla y tratar de comprenderla para asumir frente a ella una actitud adecuada.

2. Un segundo propósito de la educación política consiste en tomar conciencia de la íntima conexión que une lo político con el derecho, porque el propósito del derecho es precisamente resolver los conflictos que se presentan o se pueden presentar entre los ciudadanos de un Estado, teniendo como guía la justicia. En esa medida, cabe decir que el derecho es una de las formas de organizar la política. Esta conexión entre política y derecho implica que el objetivo fundamental de ambos debe estar orientado por la idea de justicia o, como lo ha precisado John Rawls, de la "justicia como equidad", lo que nos lleva a considerar los dos principios básicos que, al parecer del filósofo estadounidense, deben hallarse en la estructura básica de una sociedad democrática (Rawls, 1997, p. 67 ss.). Principios que determinan la asignación de derechos y deberes, de modo que las ventajas económicas y sociales se distribuyan, si no de manera igual, al menos sí de manera equitativa.

El primer principio señala que todos deben tener un igual derecho en un esquema de libertades básicas que resulte compatible con el esquema de los demás. Se trata de la elaboración de aquel precepto clásico que estipula el ejercicio de la libertad, cuyo dominio se extiende hasta donde comienza el derecho ajeno. De modo que este precepto determina el comportamiento social, ámbito, a su vez, donde se realizan y aseguran las cuatro libertades básicas, que fueron condensadas muy bien por el presidente norteamericano Franklin D. Roosevelt en su célebre Discurso del Estado de la Unión del 6 de enero de 1941: libertad de expresión, libertad religiosa, libertad de vivir sin penuria y libertad de vivir sin miedo.

Ahora bien, si el primer principio apunta a defender el ámbito de la libertad y establecer sus linderos, el segundo se refiere al manejo de las inevitables desigualdades económicas que se hacen presentes en toda sociedad: tales desigualdades deben conformarse de tal manera que resulten ventajosas para todos, con cargos y empleos asequibles. Este principio se aplica en el marco de la economía, que, si bien no puede pretender una simple igualdad en la distribución de las riquezas, debe tratar, sin embargo, de neutralizar las desventajas que surgen en el proceso de su distribución.

Si comparamos ambos principios, es clara la prioridad del primero sobre el segundo, dado que su propósito es defender las libertades básicas, cuya ausencia no 
puede ser compensada con la obtención de beneficios económicos. Sin embargo, como bien lo ha señalado Hoyos, es indispensable preservar la continuidad y la transitividad entre ambos principios, es decir, "conservar la vinculación entre los derechos primarios en torno a la libertad y los así llamados derechos humanos de 'segunda generación': los derechos sociales, económicos y culturales" (1995, p. 80 ), porque sin asegurar las condiciones básicas de equidad en el usufructo de las riquezas no es posible defender un pleno ejercicio de las libertades.

3. Un tercer propósito en la educación política consiste precisamente en aprender a conectar esos principios de carácter general con su aplicación concreta, lo que comporta no pocas dificultades. De ahí la necesidad de capacitar a los estudiantes en la toma de decisiones con la vista puesta en el bien común, de manera que activen las potencialidades presentes en los actores sociales con miras a la convivencia. Son potencialidades que hallan su campo de realización en tres ámbitos principales.

En primer lugar, en el ámbito bioético, que aborda la política desde una ética de la vida, entendida como dimensión fundamental de toda visión acerca de los espacios de actuación humana. El centro de esta perspectiva es la activación de las condiciones para una verdadera vida política, más allá de la continuidad o el simple mejoramiento circunstancial del ámbito de la supervivencia, marcado, como está, por relaciones asimétricas de poder. Si bien es cierto que las crecientes amenazas que se ciernen sobre la especie humana, derivadas de los procesos de contaminación ambiental y de sobrexplotación de los recursos naturales, exigen políticas globales por parte de los Estados, es igualmente necesario despertar y consolidar en los individuos una conciencia personal y social de responsabilidad ecológica. En efecto, no es posible olvidar que la conservación de la vida es condición sine qua non para el ejercicio de la política, de modo que no solamente todos los seres humanos nos hallamos concernidos, sino que debemos tomar conciencia de ello y aportar a su conservación en la medida de nuestras posibilidades.

En segundo lugar, más allá del marco bioético, las potencialidades humanas encuentran su lugar de realización en el ámbito intercultural, donde se articulan los diversos actores sociales y se hace presente la confrontación entre cosmovisiones contrapuestas; esta diversidad se manifiesta en las distintas valoraciones, significados y conductas que exigen ser abordados en un espacio de verdadera interlocución. Estos diálogos han de llevarse a cabo no solo globalmente, es decir, en la relación entre las diversas culturas de la humanidad actual, sino también regional y localmente, buscando promover el entendimiento y la conformación de acuerdos mínimos de respeto, valoración y aprecio de las diferencias. 
Una de las constantes preocupaciones de Hoyos, en sus intervenciones orales o escritas alrededor de la educación, giraba precisamente en torno a la impostergable necesidad de fomentar en los estudiantes una verdadera cultura del diálogo y de la discusión como el camino para buscar esos acuerdos sobre mínimos. Y llegó a bosquejar para ello una "fenomenología de lo moral" como fundamento de dichos diálogos, tomando como punto de partida los tres sentimientos morales básicos propuestos por P. F. Strawson en su ensayo sobre el problema de la libertad: de "resentimiento" ante la ofensa recibida, de "indignación" frente a la ofensa hecha a terceros y de "arrepentimiento" por la ofensa que uno ha causado (cfr. Hoyos, 1995, pp. 71-74) $)^{3}$. Sentimientos que, mediante una forma adecuada de diálogo, consideraba que podían servir de fundamento para elaborar principios mínimos de acuerdo entre cosmovisiones contrapuestas. Y citaba, a ese propósito, una acertada observación del filósofo peruano David Sobrevilla:

En lugar de proponer a todos los demás una máxima como válida y que quiero que sea ley general, tengo que presentarles a todos los demás mi máxima con el objeto de que comprueben discursivamente su pretensión de universalidad. El peso se traslada de aquello que cada uno puede querer sin contradicción como ley general, a lo que todos de común acuerdo quieren reconocer como norma universal (1987, pp. 104-105).

Finalmente, las potencialidades de los seres humanos encuentran su despliegue en el ámbito prudencial, buscando fomentar el ejercicio de una verdadera phronesis en sentido aristotélico, es decir, de una "sabiduría práctica", término griego que en español se suele traducir como "prudencia", pero cuyo alcance semántico es mucho más amplio. Se trata, en efecto, de encontrar el camino adecuado que permita avanzar, mediante la acción conjunta, hacia la obtención del mayor bien posible para la sociedad como tal, de modo que se logren respetar los dos principios básicos señalados por John Rawls y se preserve la cuádruple libertad mencionada anteriormente.

4. Un cuarto propósito de la educación política consiste en tomar conciencia del peculiar carácter temporal de lo político, cuya naturaleza se halla orientada hacia el futuro. En efecto, el propósito de la acción política es organizar las relaciones de poder de tal manera que tengan como resultado un orden social más razonable y un ejercicio más amplio de la libertad, y ello implica que, por su misma naturale-

3 Sobre el sentido de esos sentimientos morales básicos propuestos por P. F. Strawson, así como una interesante discusión sobre los mismos, puede verse McKenna \& Russell (2008). Una crítica a la propuesta de Strawson puede leerse en el artículo de Díaz (1994). 
za, toda propuesta de orden político está regida por aquello que se considera que debería ocurrir. De ahí que, en la política, el ser tenga que analizarse en función del deber-ser, es decir, que la reflexión política se configure como un esfuerzo por comprender el presente con la mirada puesta en el porvenir.

Esta orientación "futurista" del pensar y accionar político tiene su raíz antropológica en la naturaleza misma de los seres humanos, que Julián Marías (1982) ha sabido analizar con particular agudeza. Para caracterizarla, el filósofo español fraguó un neologismo muy acertado, que él mismo nos explica de la siguiente manera:

No soy futuro, entiéndase bien, sino perfectamente real y presente; pero en español hay un maravilloso sufijo: -izo, que indica inclinación, orientación o propensión; resbaladizo es aquello en que es fácil resbalar; levadizo es lo que se puede levantar; olvidadizo es el que propende a olvidar las cosas; enamoradizo, el que se enamora fácilmente; pues bien, yo soy futurizo: presente, pero orientado al futuro, vuelto a él, proyectado hacia él (p. 21).

Ahora bien, si los seres humanos somos por naturaleza "futurizos", este carácter no solo es propio de cada uno de nosotros en particular, sino que se proyecta igualmente sobre la vida en comunidad, de tal manera que bien cabe afirmar que las sociedades tienen una naturaleza igualmente "futuriza", volcada hacia lo que ha de venir. Esta característica de los seres humanos y las sociedades es la que nos permite entender por qué la reflexión y la acción política buscan precisamente configurar aquello que está por venir o que se halla en el porvenir.

De ahí que, al elaborar un proyecto político o al pretender su instauración, nos estemos moviendo inevitablemente en el campo de las suposiciones, de las conjeturas, es decir, de aquello que consideramos como posiblemente verdadero, incluso como lo deseablemente verdadero, pero cuya verdad nunca podrá ser demostrada a priori, es decir, antes de pasar por la experiencia. En el pensamiento y la acción política nos movemos en el ámbito de aquello que los escolásticos llamaron los "futuribles" y que el Diccionario de la Lengua define muy bien como "una cosa que podría existir o producirse en el futuro, especialmente si se diese una condición determinada".

Fuerza es recordar aquí la admonición que hace Hegel, al final del Prefacio a las Lineas fundamentales de la Filosofía del Derecho, dirigida a los filósofos en su tarea de perseguir la verdad y orientar el comportamiento: 
Para decir una palabra acerca de la pretensión de enseñar (Belehren) cómo debe ser el mundo, la filosofía, en todo caso, llega en ello siempre demasiado tarde. Como pensamiento del mundo, ella viene a aparecer en el tiempo después de que la realidad ha completado su proceso cultural y lo ha llevado a término. [...]. Cuando la filosofía dibuja su gris sobre el gris, es porque una figura de la vida ha envejecido, y con el gris sobre gris no se la puede rejuvenecer, sino únicamente reconocer; sólo cuando irrumpe el ocaso inicia su vuelo el búho de Minerva (Hegel, 1955, p. 17).

En ese mismo sentido, si bien desde una perspectiva muy diferente, se expresa Baruch Spinoza en su Tratado politico, cuando dice que los filósofos

[...] conciben a los seres humanos no como son, sino como ellos quisieran que fuesen. De ahí que las más de las veces hayan escrito una sátira en vez de una ética, y que no hayan ideado jamás una política que pueda llevarse a la práctica, sino otra que, o debería ser considerada como una quimera, o solo podría ser instaurada en el país de la Utopía o en el siglo dorado de los poetas, es decir, allí donde no hacía falta alguna (Spinoza, 1986, I, \$1, p. 78).

Ambos pensadores coinciden en señalar que el campo de la política, estrictamente hablando, no pertenece al dominio de la filosofía, porque se trata del ámbito de lo verosímil, lo probable, lo conjeturable, mientras que la intención de la filosofía es buscar lo verdadero, lo necesario, es decir, no solo lo que es, sino lo que ha tenido que ser. De ahí que el ejercicio tanto teórico como práctico de la política debe conllevar una alta dosis de escepticismo, ajeno a todo dogmatismo, sin que ello signifique, por supuesto, un escepticismo con respecto a los valores fundamentales que hay que defender, sino en aquello que hace referencia a los caminos para lograrlo. Ello es así porque en los programas políticos se trata siempre, y de manera inevitable, de propuestas más o menos plausibles, más o menos convincentes, pero sometidas todas ellas al juicio inexorable de la realidad, ya que se despliegan en el ámbito de lo que consideramos que debería ser. Solo así podemos entender que la divergencia de opiniones políticas, en el marco de un reconocimiento pleno de los valores fundamentales, en vez de ser un fenómeno que deberíamos buscar suprimir o neutralizar, es en realidad un elemento fundamental del juego democrático, porque de él se deriva la riqueza en la búsqueda del mejor camino.

Ahora bien, esta naturaleza hipotética o futurible de todo proyecto político proviene no solo del carácter "futurizo" tanto de los individuos como de las sociedades que ellos conforman, sino también de que su realización debe pasar de manera inexorable por la rigurosa criba de la convicción, si queremos salvaguardar un orden democrático; en otras palabras, no basta con que los argumentos que lo 
sustentan sean sólidos, sino que tienen que llegar a ser convincentes. Y esto implica pasar de la lógica general a la lógica retórica, que no obedece a criterios meramente formales, ya que su propósito fundamental no es demostrar, sino convencer ${ }^{4}$.

De ahí que, si bien es cierto que la realidad política, como hemos visto, tiene el carácter de lo ineludible, se orienta además a resolver los conflictos sociales a la luz de la justicia y se despliega necesariamente en el ámbito de lo futurible, es decir, de lo hipotético; cabe muy bien, respecto a ella, glosar la amonestación a los creyentes que Anselmo de Canterbury puso en labios de su contertulio Boso: "considero que es negligencia si después de que hemos sido confirmados en la fe, no tratamos de entender aquello que creemos" (Anselmo, 1952, I, p. 2). Consideramos que es negligencia si, una vez formamos parte de una sociedad democrática, no tratamos de entender la forma como se ejerce en ella el poder y se posibilita la libertad, es decir, si no nos esforzamos por comprender nuestra función política.

\section{Escollos que deberán evitarse}

Luego de señalar la importancia que tiene la formación política de los estudiantes universitarios y de esbozar algunos de sus principales propósitos, es bueno considerar también los escollos que un proyecto como este deberá sortear, porque el campo de la política suele estar marcado por sentimientos, juicios de valor y convicciones personales que pueden llegar a distorsionar el proceso educativo, cuyo objetivo primordial debe estar dirigido al ejercicio de las condiciones para un verdadero diálogo entre quienes sostienen posturas divergentes.

1. El primer escollo, y tal vez el más peligroso, ya ha sido indicado cuando hicimos alusión a la opinión expresada por Guillermo Hoyos: hay que evitar que la cátedra académica se convierta en una tribuna para el proselitismo ideológico o partidista. No se puede confundir lo político, o la política tout court, es decir, la política sin más, y la política partidista. Ambas son válidas, más aún, ambas son indispensables en una sociedad democrática, pero pertenecen a ámbitos distintos y no se deben confundir.

En realidad, este es un peligro en el que puede caer fácilmente un proyecto de educación política, ya que, si todo ciudadano debe interesarse y participar, en la medida de sus posibilidades y en el contexto de su situación, en el juego de la po-

4 A este propósito puede consultarse el artículo de Gómez (2001). 
lítica es normal que quien educa tenga sus propias preferencias partidistas o ideológicas. Y no se puede pretender que los docentes sean "eunucos" políticos, así como tampoco pueden convertirse en simples agitadores en favor de una causa.

No se puede olvidar que una las grandes virtudes de un buen educador consiste precisamente en lograr que sus estudiantes desarrollen su propia manera de pensar, de modo que la bien conocida consigna ilustrada de Kant, "Sapere aude" ("Atrévete a pensar"), si bien es cierto que se dirigía en primer lugar a cada uno de sus lectores para aguijarlos a ejercer su libertad de pensamiento, conlleva igualmente, por parte de quienes ejercen la docencia, la obligación de respetar e incentivar dicha libertad en sus alumnos. Más que trasmitirles las propias conclusiones o convicciones, logradas como fruto de la reflexión personal del docente, su deber es motivarlos para que ellos, mediante un ejercicio adecuado y consistente de su capacidad reflexiva, elaboren sus propias convicciones personales.

No se trata, por supuesto, de tener que ocultar las convicciones políticas o de hablar y obrar como si no se las tuviera; una actitud así, cuando va más allá de los límites que señala la prudencia, termina convirtiéndose en hipocresía o pusilanimidad. Se trata, más bien, de tener muy claro que las convicciones políticas, por "claras y distintas" que le parezcan a quien las tiene, no pasan de ser más que interpretaciones hipotéticas sobre cómo se debería organizar la sociedad para lograr un futuro mejor. No se puede olvidar, como lo hemos seńalado anteriormente, que el ámbito de lo político se despliega en el marco de lo presumible y no puede aspirar nunca a convertirse en verdad "monda y lironda". En la raíz misma de una verdadera sociedad democrática debe estar muy claro, para todos los que participan en ella, que la defensa de las convicciones políticas nunca debe olvidar que se trata de hipótesis más o menos verosímiles, pero nunca de verdades absolutas. Olvidarlo significa caer en el fanatismo político.

2. Ahora bien, un segundo escollo que debe sortear una educación política es el de caer en un falso maniqueísmo, escollo que está muy ligado con el anterior. Es que en el análisis de las posiciones políticas nada parece más dañino que las divisiones dicotómicas y si caracterizamos esas divisiones con los términos de "verdad" y "error", la consecuencia será que quienes sustentan una posición política se creen en posesión de la verdad, mientras que sus adversarios se hallan sumidos en el error. Y resulta muy fácil pasar de ese erróneo juicio epistémico a formular un juicio moral, porque, en principio, el error no tiene derechos, de modo que quienes se creen en posesión de la verdad serán los buenos y quienes parecen empecinarse en el error serán los malos. 
No se puede negar que tales divisiones suelen ofrecer una ventaja retórica nada despreciable, ya que permiten diferenciar con facilidad aquello que en la realidad es muy complejo. Pero esa misma ventaja retórica tiene efectos deplorables para la comprensión de lo político, ya que detrás de tales divisiones dicotómicas se encuentra siempre larvada la división maniquea entre el bien y el mal. Cuando diferenciamos las posiciones políticas bajo el fácil expediente de una derecha y una izquierda, por ejemplo, de tal manera que si alguien o alguna posición política no puede considerarse de izquierda tiene que calificarse necesariamente como de derecha, o viceversa, es muy fácil que una diferenciación tan simple se deslice de manera imperceptible hasta convertirse en una calificación moral, en una diferenciación entre buenos y malos. Y, por supuesto, siempre nos creeremos situados del lado de los buenos, de modo que quienes no comparten nuestras convicciones políticas terminan perteneciendo al grupo abominable de los malos.

$\mathrm{Si}$, a manera de ejemplo, nos fijamos en los procesos políticos en las sociedades democráticas, podemos decir, en una visión muy esquemática, pero tal vez por ello mismo esclarecedora, que todas las propuestas políticas comparten al menos tres valores básicos para la conformación de una sociedad ideal, a saber, el orden, la libertad y la justicia. Todas están de acuerdo en que una sociedad en la que reinaran esos tres valores estaría muy cerca de ser una sociedad ideal. El problema consiste en que tales valores no parece que puedan lograrse plenamente de manera simultánea y esto exige, entonces, que se establezca una jerarquía entre los mismos.

De manera muy esquemática, puede decirse que, para los llamados conservadores, el orden es considerado el valor que condiciona a los otros dos, porque estiman indispensable salvaguardar el orden si se quiere llegar a una sociedad en verdad libre y justa. Quienes se proclaman liberales, por su parte, consideran que es la libertad la que tiene el carácter de conditio sine qua non, porque sin libertad el orden aparece como sometimiento, a la vez que desaparece la justicia. Las corrientes marxistas, finalmente, estiman que, para alcanzar un verdadero orden y una verdadera libertad, es necesario comenzar por establecer la justicia, incluso sacrificando en parte el orden y postergando un tiempo el ejercicio pleno de la libertad.

Entonces, la pregunta sería: ¿una visión así, tripartita y no dicotómica, no nos permitiría comprender mejor el juego de la política? Con ello se evita establecer a priori una línea divisoria entre la verdad y el error, y convertirla luego en otra entre el bien y el mal. Y tendría, además, la ventaja de permitir pensar que cada una de tales posiciones puede pretender ser preferible, de acuerdo con el contexto 


\section{Jorge-Aurelio Díaz, Darwin Muñoz y Edgar Javier Garzón-Pascagaza Educación, política y universidad}

y la coyuntura de una sociedad. Porque si, como hemos visto, la política se halla proyectada hacia el futuro, no puede olvidar que, con el paso del tiempo, las condiciones suelen cambiar de manera muy significativa. De modo que si la política no se mueve en el ámbito de la verdad, sino de las hipótesis, quienes participan en ella deben mantener abierta su mente para cambiar sus opiniones cuando así lo exijan las cambiantes circunstancias. Lejos de todo dogmatismo, la democracia exige mentes abiertas y flexibles.

3. Un tercer escollo puede derivarse de aquellos profesores que, en virtud de la libertad de cátedra, pretendan convertirla en tribuna política, abusando de la autoridad que les confiere su calidad de maestros. Para evitar este escollo, conviene traer a colación una importante observación de Kant en su conocido escrito Respuesta a la pregunta: ¿qué es la Ilustración? Cabe recordar que el filósofo de Königsberg es reconocido como uno de los grandes defensores de la libertad humana.

Luego de definir la Ilustración como "la salida del hombre de su condición de menor de edad de la cual él mismo es culpable" (2002, A 481, p. 5)5, Kant señala que "para esa Ilustración solo se exige libertad y, por cierto, la más inofensiva de las que pueden llamarse libertad, a saber, la libertad de hacer uso público de la propia razón en todo respecto" (A 485, p. 7). Sin embargo, acepta que en determinadas ocasiones este uso puede ser restringido, y se pregunta: “¿qué limitación impide la Ilustración y cuál, por el contrario, la fomenta?” (p. 7), a lo que responde con una importante distinción: "el uso público de la razón debe ser libre siempre, y es el único que puede producir la ilustración entre los hombres. El uso privado de la misma, en cambio, debe ser con frecuencia severamente limitado, sin que obstaculice con ello particularmente el progreso de la ilustración” (p. 7). Y pasa a explicar la diferencia: "Entiendo por uso público de la propia razón el que alguien hace de ella en cuanto que sabio ante la totalidad del público rector. Llamo uso privado al empleo de la razón que se le permite al hombre en el interior de una posición civil o de una función que se le ha confiado" (p. 7).

Por consiguiente, si los profesores desempeñan funciones en servicio de la comunidad, deben tomar conciencia de que, siguiendo la distinción de Kant, el uso de su razón no es "público", sino "privado", y se halla restringido en beneficio de la misma ilustración, ya que no pueden pretender que sus estudiantes terminen compartiendo sus personales convicciones. En lugar de proponerse "adoctrinar",

5 Se indica la paginación de la edición de la Academia y la de la traducción al español. 
el profesor debe "ilustrar", es decir, ofrecerles a los alumnos las mejores condiciones para que ellos mismos elaboren sus propias convicciones políticas.

\section{Conclusiones}

A manera de conclusión y retomando una vez más la insinuación hecha en su momento por el profesor Guillermo Hoyos -a quien hemos querido tributar un reconocimiento como verdadero maestro-, consideramos necesario que las universidades reflexionen seriamente sobre el compromiso que tienen para con la sociedad de participar activamente en la formación de ciudadanos conscientes y responsables. Es cierto que, con el cabal cumplimiento de sus funciones docentes y de investigación, las instituciones de educación superior cumplen ya una meritoria tarea social de innegable valor. Pero en el seno de una sociedad democrática se hace necesario que las universidades den un paso más y establezcan las condiciones y los espacios necesarios para que sus estudiantes, y también sus profesores, tengan la oportunidad de reflexionar seriamente sobre sus derechos y deberes como ciudadanos, y sobre la importancia que para ello tienen los procesos políticos. Si no se logra que los ciudadanos, cada uno desde su lugar y con sus posibilidades, asuman una posición consciente sobre sus derechos y sus deberes en el campo de lo político, se corre el peligro de ver cómo se debilita la democracia y con ello se cierne una grave amenaza sobre los derechos de todos, porque la responsabilidad política recae sobre todo y de manera creciente en quienes han tenido la oportunidad de recibir una educación superior; ya que, si bien es cierto que la educación aumenta de manera significativa los derechos al abrir nuevos horizontes de realización, también lo es que a todo derecho corresponde un deber, de modo que al acrecentarse los derechos se acrecientan en igual medida los deberes.

\section{Referencias}

Anselmo de Canterbury (1952). Obras Completas I. Madrid: Biblioteca de Autores Cristianos.

Arendt, H. (2008). La promesa de la política. Barcelona: Editorial Paidós.

Díaz, J.-A. (1994). El resentimiento como argumento moral. Ideas y Valores, 43(94), pp. 11-20.

Durán, V. (ed.) (2013). Guillermo Hoyos: vida y legado. Bogotá: Pontificia Universidad Javeriana. 
54 | Jorge-Aurelio Díaz, Darwin Muñoz y Edgar Javier Garzón-Pascagaza Educación, política y universidad

Gaitán-Riveros, C. A. \& Martínez-Posada, J. E. (eds.) (2013). Universidad y sociedad: aproximaciones críticas, tensiones y desafíos. Bogotá: Pontificia Universidad Javeriana.

Garzón, E.-J. (2016). Notas sobre política en el pensamiento de Hannah Arendt: vitalidad de la acción humana. En P. Cardona-Restrepo et al. (eds.), El filósofo y lo político: autores contemporáneos (pp. 45-55). Medellín: Universidad Pontificia Bolivariana.

Gómez, A.-L. (2001). Tres aproximaciones a la Teoría de la argumentación de PerelmanOlbrechts. Revista Lenguaje, 28, pp. 9-17.

Hegel, G. W. F. (1955). Grundlinien der Philosophie des Rechts. Hamburg: Verlag von Felix Meiner.

Hoyos, G. (2013). Filosofía de la educación. Apuntes de su último seminario de doctorado. Bogotá: Siglo del Hombre Editores - Universidad Tecnológica de Pereira.

_ (1995). Ética comunicativa y educación para la democracia. Revista Iberoamericana de Educación, 7, pp. 65-91.

_ (2012). Ensayos para una teoría discursiva de la educación. Bogotá: Editorial del Magisterio.

Kant, Immanuel (1964). Kant Werke, Band 9. Darmstadt: Wisseenschaftliche Buchgesellschaft.

_ (2002). Respuesta a la pregunta: ¿qué es la llustración? Trad. Rubén Jaramillo. Bogotá: Universidad Nacional de Colombia, Facultad de Ciencias Humanas.

Marías, J. (1982). Antropología filosófica. Ensayos. Madrid: Revista de Occidente.

McKenna, M. \& Russell, P. (eds.) (2008). Free Will and Reactive Attitudes. Perspectives on P. F. Strawson's "Freedom and Resentment". Surrey: Ashgate Publishing Limited.

Nussbaum, M. (2010). Sin fines de lucro. Por qué la democracia necesita de las humanidades. Trad. María Victoria Rodil. Barcelona: Editorial Katz.

Rawls, J. (1997). Teoría de la Justicia. Trad. María Dolores González. México: Fondo de Cultura Económica.

Roosevelt, F. D. (1941). The Four Freedoms. Recuperado de http://www.americanrhetoric.com/speeches/PDFFiles/FDR\%20-\%20Four\%20Freedoms.pdf.

Sobrevilla, D. (1987). El programa de fundamentación de una ética discursiva de Jürgen Habermas. Ideas y Valores, 36(74-75), pp. 99-117.

Spinoza, B. (1986). Tratado político. Trad. Atilano Domínguez. Madrid: Alianza Editorial.

Strawson, P. F. (1995). Libertad y resentimiento y otros ensayos. Trad. Juan José Acero. Barcelona: Editorial Paidós.

Vargas, G.; Meléndez, R. \& Herrera, W. (2017). Experiencia y problemas. Educación ciudadana y enseñanza de la filosofía. Pedagogía y Saberes, (47), pp. 65-77.

Vasco, C. E.; Vasco, E. \& Ospina, H. F. (eds.) (2009). Ética, política y ciudadanía. Bogotá: Siglo del Hombre Editores, Universidad de Manizales, Centro Internacional de Educación y Desarrollo Humano (CINDE). 